\title{
COMPUESTOS VOLÁTILES RESPONSABLES DEL SABOR DEL TOMATE
}

\author{
VOLATILE COMPOUNDS RESPONSIBLE OF TOMATO FLAVOR
}

\author{
Jorge E. de J. Dávila-Aviñaa ${ }^{1}$, Gustavo A. González-Aguilar², Jesús F. Ayala-Zavala ${ }^{2}$, David R. Sepúlveda ${ }^{1}$ y \\ Guadalupe I. Olivas ${ }^{1 \star}$
}

\begin{abstract}
${ }^{1}$ Laboratorio de Tecnología de Alimentos de Origen Vegetal, Centro de Investigación en Alimentación y Desarrollo, A. C. Av. Río Conchos s/n, Parque Industrial. 31570, Cuauhtémoc, Chihuahua, México. Tel.: 01 (625) 5812921 ext. 118; Fax 01 (625) 5812921 ext. 113. ${ }^{2}$ Dirección de Tecnología de Alimentos de Origen Vegetal, Centro de Investigación en Alimentación y Desarrollo, A. C. Carretera a la Victoria km. 0.6. Apartado Postal 1735. 83000, Hermosillo, Sonora, México.

* Autor para correspondencia (golivas@ciad.mx)
\end{abstract}

\section{RESUMEN}

La producción de frutos de tomate (Lycopersicon esculentum Mill.) con sabor agradable está recibiendo atención especial, porque este atributo se ha convertido en uno de los principales parámetros de calidad en la aceptación de frutas y hortalizas. Aquí se analiza cómo los compuestos volátiles influyen significativamente en el sabor del tomate, por ser responsables de su aroma. Los principales compuestos volátiles del tomate involucran a aldehídos, alcoholes, cetonas y ésteres insaturados de cadenas cortas, los cuales son producidos a partir de ácidos grasos, aminoácidos libres y carotenoides. Entre las principales enzimas involucradas se encuentran lipoxigenasa, hidroperoxidoliasa y alcohol deshidrogenasa para la biosíntesis de volátiles a partir de ácidos grasos; además están las enzimas aminotransferasa, descarboxilasa y alcohol deshidrogenasa, relacionadas con la biosíntesis de volátiles derivados de aminoácidos. La composición y concentración de los compuestos volátiles depende de factores genéticos, así como de las condiciones de cultivo y del almacenamiento. El presente manuscrito compila y analiza la información actual referente a la biosíntesis de los volátiles que definen el aroma del tomate, así como sus rutas metabólicas, enzimas y sustratos involucrados; así mismo analiza los principales factores precosecha, cosecha y poscosecha que afectan su biosíntesis.

Palabras clave: tomate, Lycopersicon esculentum, aroma, compuestos volátiles, calidad de fruto.

\section{SUMMARY}

Production of tomato fruits (Lycopersicon esculentum Mill.) with good flavor is receiving special attention since flavor has become a primary quality parameter in the acceptance of fruits and vegetables. In this manuscript we analyze how volatile compounds play a significant role in flavor perception of tomatoes, as they are responsible of their aroma. The most important volatile compounds in tomato include short-chain unsaturated aldehydes, alcohols, ketones and esters which are synthesized from free amino acids, fatty acids and carotenoids. Among the main enzymes involved in the biosynthesis of volatiles derived from fatty acids are lipoxygenase, alcohol dehydrogenase and hydroperoxide lyase, while aminotransferase, decarboxylase and alcohol dehydrogenase are involved in the biosynthesis of volatiles derived from amino acids. This manuscript brings together the current information related to tomato volatiles biosynthesis, including metabolic pathways, enzymes and substrates. In addition, the effects of preharvest conditions and postharvest handling on volatile production are also discussed.

Index words: Lycopersicon esculentum, aroma, volatile compounds, fruit quality.

\section{INTRODUCCIÓN}

El tomate o jitomate (Lycopersicon esculentum Mill.) es uno de los cultivos más importantes en México (GarcíaSahagún et al., 2009). Después de la papa (Solanum tuberosum L.), el tomate es la hortaliza de la familia de la Solanáceas más cultivada en el mundo (Bombelli y Wright, 2006) y uno de los vegetales más consumidos por su versatilidad, sus propiedades nutrimentales y su sabor característico (Lewinsohn et al., 2001; Sinesio et al., 2010).

El sabor juega un papel central en la calidad del tomate porque determina la aceptación del consumidor, pues si bien la decisión inicial de compra se basa en la apariencia, las adquisiciones posteriores dependerán principalmente de la evaluación del sabor que el comprador realice al consumirlo (Sinesio et al., 2010). En décadas recientes, la falta de sabor del tomate comprado en supermercados ha sido una queja común del consumidor (Ortiz-Serrano y Gil, 2010). La deficiencia en el sabor del tomate es, en gran medida, debida al desconocimiento y escaso control del metabolismo de la producción de compuestos volátiles, y al desarrollo de nuevas variedades más resistentes o firmes, pero con una reducida producción de compuestos del sabor (Baldwin et al., 2000). Es entonces necesario un mayor entendimiento de la biosíntesis de los componentes del sabor del tomate, para lograr un mejor control de este importante atributo de calidad. 
El sabor del tomate está definido por los azúcares (fructosa y glucosa), aminoácidos libres (glutamato) y ácidos orgánicos (ácido cítrico), pero en mayor medida por la amplia variedad de compuestos volátiles que proporcionan su aroma (Maneerat et al., 2002; Anza et al., 2006; Narain et al., 2010; Sorrequieta et al., 2010).

Se han identificado más de 400 compuestos volátiles en el fruto de tomate, de los cuales sólo 30 presentan concentraciones por encima de una parte por billón (ppb); de éstos, sólo unos cuantos compuestos contribuyen mayormente a la percepción del aroma (Baldwin et al., 2000; Buttery y Ling, 1993), pero es difícil relacionar el aroma del tomate con un compuesto en específico (Lewinsohn et al., 2001). La biosíntesis de estos compuestos volátiles abarca rutas bioquímicas que involucran diferentes enzimas y sustratos, donde los ácidos grasos, aminoácidos libres y carotenoides son los principales precursores (Tieman et al., 2007). La composición y concentración de los compuestos volátiles puede intensificar la percepción del sabor del tomate o contribuir a la formación de sabores desagradables; estas concentraciones pueden variar según la variedad, el estado de madurez, el periodo de almacenamiento y los tratamientos postcosecha (Boukobza y Taylor, 2002; Krumbein et al., 2004; Tandon et al., 2003).

En este contexto, el objetivo de esta revisión es compilar y analizar la información actual disponible sobre los compuestos volátiles responsables del aroma del tomate, con énfasis en su composición y síntesis, así como el efecto que provocan diferentes factores pre y postcosecha sobre el aroma de estos frutos y su aceptación por parte del consumidor.

\section{IMPORTANCIA DEL AROMA EN EL SABOR DEL FRUTO DE TOMATE}

El sabor característico del tomate está dado por la interacción entre ácidos orgánicos, azúcares y compuestos volátiles (Baldwin et al., 2008). Los ácidos orgánicos y azúcares se perciben mediante el sentido del gusto, donde son disueltos en la saliva e interactúan con los receptores del gusto (microvellosidades) al causar cambios eléctricos en las células y enviar señales al cerebro donde se transformarán para desencadenar una sensación de sabor (Beullens et al., 2008).

La percepción del aroma del tomate depende de la composición y concentración de los compuestos volátiles que estimulan a las neuronas olfativas ubicadas en el epitelio olfatorio en el techo de la cavidad nasal. Los pasos involucrados en la percepción del aroma son: masticación del fruto, liberación del aroma, percepción del mismo por los receptores olfatorios, y envío de la información al cerebro donde el bulbo olfativo procesa todas las señales del epitelio olfatorio y crea una sensación de aroma (AyalaZavala et al., 2009; Taylor et al., 2006).

Las sustancias no volátiles como azúcares, sales y ácidos orgánicos sólo estimulan el sentido del gusto, mientras que las sustancias volátiles lo hacen tanto en el gusto como en el olfato. Si bien estas moléculas se encuentran en muy baja concentración en el tomate, su contribución en el aroma y sabor es muy importante (Tieman et al., 2007; Taylor, 2002).

\section{PRINCIPALES COMPUESTOS VOLÁTILES DEL AROMA DEL FRUTO DE TOMATE}

En general todas las frutas y hortalizas producen un conjunto de compuestos de bajo peso molecular que poseen cierta volatilidad a temperatura ambiente. Estos compuestos pueden no tener importancia cuantitativa, pero definen el aroma de frutas y hortalizas (Kader, 2008). El sabor único de los tomates frescos está determinado por un grupo de volátiles presentes en ciertas proporciones.

Los más importantes compuestos volátiles responsables del sabor del tomate incluyen: aldehídos insaturados de cadena corta, alcoholes (C3-C6), cetonas y ésteres (Cuadro 1; Tikunov et al., 2005). Su biogénesis ocurre durante el desarrollo de la madurez y se incrementa en el pico climatérico. Los aromas típicos del tomate durante su maduración en campo son atribuidos en 58 $\%$ a ésteres y alcoholes de cadena larga, $32 \%$ a cetonas y aldehídos y $10 \%$ a alcoholes de cadena corta (Eskin, 1979). Tikunov et al. (2005) dividen a los principales compuestos volátiles del tomate en los siguientes grupos: a) volátiles fenilpropanoides; b) otros fenólicos volátiles; c) derivados de leucina e isoleucina; d) derivados de lípidos; e) terpenoides; f) derivados de carotenoides de cadena abierta; y g) derivados de carotenoides cíclicos.

La percepción del aroma del tomate se debe principalmente a la combinación de cis-3-hexenal, hexanal, trans-2-hexenal, hexanol, cis-3-hexenol, 2-isobutiltiazol, 6-metil-5-hepten2-ona, geranilacetona, feniletanol, $\beta$-ionona, 1 -penten-3ona, 3 -metilbutanol, 3-metilbutanal y 2-pentenal (Buttery y Ling, 1993; Malundo et al., 1995), además de salicilato de metilo, pentanal, acetona, etanol, metanol y furanol, que en concentraciones adecuadas producen el aroma fresco del tomate durante la maduración (Tandon et al., 2000). Los volátiles hexanal, cis-3-hexenal, cis-3-hexenol, trans-2hexenal y 2 -isobutiltiazol, son compuestos importantes que potencian el aroma verde característico del tomate (Yilmaz et al., 2002a). 
Cuadro 1. Clasificación de 70 compuestos volátiles responsables del aroma en tomate (Fuente: Tikunov et al., 2005).

\begin{tabular}{|c|c|c|c|c|}
\hline \multicolumn{2}{|c|}{ Derivados de Lípidos } & $\begin{array}{c}\text { Volátiles } \\
\text { fenilpropanoides }\end{array}$ & \multicolumn{2}{|c|}{ Otros fenólicos volátiles } \\
\hline 1-Penten-3-ol & 2-Hexenal & Salicil aldehído & Tolueno & 2-Fenil-3-buten-ol \\
\hline 1-Penten-3-ona & E-2-Hexenal & Guaiacol & Etilbenceno & Alcohol bencílico \\
\hline Pentanal & Z-3-Hexenol & Salicilato de metilo & Estireno & Fenil acetaldehído \\
\hline 2-Etilfuran & 1-Hexenol & Salicilato de etilo & 1-Fenilpropano & $\rho$-Cresol \\
\hline E-2-Pentenal & Heptanal & Eugenol & Benzaldehído & a-Fenilpropionaldehído \\
\hline 1-Pentanol & E,E-2,4-Hexadienal & & Fenol & Feniletanol \\
\hline Z-2-Penten-1-ol & E-2-Heptenal & & $\rho$-Metilestireno & Fenilacetonitrilo \\
\hline Z-3-Hexenal & 2-n-Pentilfuran & & Benzonitrilo & $\beta$-Fenilpropionaldehído \\
\hline Hexanal & E,E-2,4-Heptadienal & & & \\
\hline \multicolumn{2}{|c|}{ Derivados de Leucina e Isoleucina } & Terpenoides & $\begin{array}{l}\text { Derivados de carotenos } \\
\text { (cadena abierta) }\end{array}$ & $\begin{array}{c}\text { Derivados de carotenos } \\
\text { (cíclicos) }\end{array}$ \\
\hline \multicolumn{2}{|c|}{ 3-Metilbutanal } & Limoneno & 6-Metil-5-hepten-2-ona & $\alpha$-Isoforona \\
\hline \multicolumn{2}{|r|}{ 2-Metilbutanal } & Linalol oxido, Z- & 6-Metil-5-hepten-2-ol & Acetofenona \\
\hline \multicolumn{2}{|r|}{ 3-Metilbutanol } & Linalol oxido, E- & 5-Hexen-2-ona,5-metil-3- & $\beta$-Ciclocitral \\
\hline \multicolumn{2}{|r|}{ 2-Metilbutanol } & Ocimenol & metileno & $\beta$-Damascenona \\
\hline \multicolumn{2}{|r|}{ E-2-Metil-2-butenal } & $\rho$-Cimen-8-ol & 6-Metil-3,5-heptadien-2-ona & $\beta$-Ionona \\
\hline \multicolumn{2}{|r|}{ 3-acido Metilbutanoico } & Acetofenona,4-metil & $\beta$-Citral & \\
\hline \multicolumn{2}{|r|}{ 3-Metilbutanol nitrito } & a-Terpineol & a-Citral & \\
\hline \multicolumn{2}{|r|}{$\mathrm{C}_{5} \mathrm{H}_{9} \mathrm{NO}_{2}$} & 2-Caren-10-al & Geranil acetona & \\
\hline \multicolumn{2}{|r|}{$\mathrm{C}_{5} \mathrm{H}_{11} \mathrm{NO}_{2}$} & & Pseudoionona & \\
\hline \multicolumn{2}{|r|}{ 2-Isobutiltiazol } & & & \\
\hline
\end{tabular}

Los compuestos percibidos por el consumidor difieren de los compuestos encontrados en el fruto intacto, ya que durante la masticación se rompen los tejidos y quedan expuestos sustratos y enzimas que conducen a una rápida generación de compuestos volátiles en la boca (Piggott y Schaschke, 2001). Según Yilmaz (2001a), durante el daño celular provocado por maceración o cortado del tomate, se producen cambios significativos en el sabor del fruto, ocasionados por la aparición o incremento en la concentración de volátiles como cis-3-hexanal, trans2-hexenal, hexanal, trans-2-heptenal, 1-penten-3-ona, 1-penten-3-ol, geranilacetona y trans-2-pentanal. En cambio los volátiles 3-metilbutanol, pentanol, cis-3hexenol, hexanol, 6-metil-5-hepten-2-ona, fenilaldehído, 2-feniletanol, 2-isobutiltiazol y 1-nitro-2-feniletano no muestran cambios significativos cuando el tejido es macerado (Yilmaz, 2001a).

\section{BIOSÍNTESIS DE COMPUESTOS VOLÁTILES DEL AROMA EN EL FRUTO DE TOMATE}

La generación de compuestos volátiles en tomate es un proceso complejo en el que participan enzimas y sustratos dependientes del estado de madurez del fruto, y que a través de diferentes vías metabólicas conducen a la biosíntesis de estos compuestos (Cuadro 2). La producción de volátiles es afectada por condiciones celulares como: disponibilidad de substratos y enzimas, ubicación de las reacciones, propiedades físicas de los sustratos y actividad de las enzimas (Schwab et al., 2008). Los principales sustratos de estos volátiles son los aminoácidos libres, ácidos grasos y carotenoides (Baysal y Demirdöven, 2007; Tieman et al., 2007). Entre las enzimas de mayor importancia en la síntesis de volátiles se encuentran lipoxigenasa (LOX),

\begin{tabular}{cccc}
\multicolumn{2}{c}{ Cuadro 2. Principales compuestos volátiles del tomate y sus precursores. (Tandon et al., 2000). } \\
\cline { 1 - 2 } Derivados de lípidos & Derivados de aminoácidos & & Derivados de carotenoides \\
\hline Hexanal & Leucina: & & B-Caroteno: \\
Hexanol & 3-Metilbutanol & & B-Ionona \\
Cis-3-Hexanal & 3-Metilbutanal & & \\
Trans-2-Hexanal & 2-Isobutiltiazol & & \\
Cis-3-Hexenol & & & Fitoeno: \\
Pentanal & Fenilalanina: & & Geranilacetona \\
\cline { 2 - 3 } & 2-Feniletanol & & Licopeno: \\
& & & 6-Metil-5-Hepten-2-ona \\
\hline
\end{tabular}


hidroperóxido-liasa (HPL) y alcohol deshidrogenasa $(\mathrm{ADH})$, presentes en la oxidación de lípidos; así como aminotransferasa, descarboxilasa y $\mathrm{ADH}$, relacionadas con la biosíntesis de volátiles derivados de aminoácidos (Peach et al., 2008).

\section{Compuestos volátiles formados por degradación de ácidos grasos}

Los compuestos volátiles derivados de ácidos grasos son los principales contribuyentes al aroma del tomate (Yilmaz, 2001b). La Figura 1 muestra las reacciones que se llevan a cabo para la formación de estos volátiles. Oxipilina es el nombre genérico de la familia de compuestos oxigenados formados por ácidos grasos mediante reacciones enzimáticas, en las cuales participan las enzimas LOX, HPL y ADH. Algunas isoenzimas pueden oxidar algunos lipoglicéridos pero, en general, los sustratos de la LOX son los ácidos grasos libres (Schwab y Schreier, 2002), principalmente los ácidos linoleico y linolénico (Chen et al., 2004).

La primera enzima involucrada en la producción de volátiles a partir de ácidos grasos es la LOX. Los productos de las reacciones de la LOX dependen del estímulo natural de la célula, de la disponibilidad de sustratos y de los sitios intracelulares donde se llevan a cabo las reacciones (Gargouri et al., 2008). Se conocen a la fecha cinco lipoxigenasas: TomloxA, TomloxB, TomloxC, TomloxD y TomloxE; el rol específico de cada una de ellas es todavía incierto (Chen et al., 2004), y cada una puede tener funciones distintas en la biosíntesis de compuestos volátiles durante el desarrollo del fruto. Se conoce que el gen de TomloxD se expresa en bajo grado en frutos de tomate en estado verde-maduro y maduro, mientras que TomloxC se expresa durante la maduración y en el pico climatérico; la expresión de TomloxB se incrementa conforme aumenta la producción de etileno, mientras que la de TomloxA disminuye (Heitz et al., 1997; Griffiths et al., 1999). La catálisis de la LOX consiste de tres fases: eliminación de un hidrógeno estereoespecífico del grupo metileno entre dos dobles enlaces, reordenamiento del radical libre resultante, y vinculación del oxígeno molecular al radical libre (Maróstica Jr y Pastore, 2007).

Unos estudios han revelado un incremento lineal en la actividad de la LOX durante la maduración de los frutos de tomate, cuya actividad máxima ocurre en el estado quebrante (cambio de color verde a rojo) del fruto, y luego disminuye en el estado maduro (Yilmaz et al., 2001). A nivel celular la actividad de la LOX en frutos de tomate se ha reportado tanto en el sistema membranoso como en la parte soluble, y su mayor actividad se lleva a cabo entre la epidermis y la carnosidad del fruto (Yilmaz, 2001b).

El mecanismo de la enzima HPL involucra varias

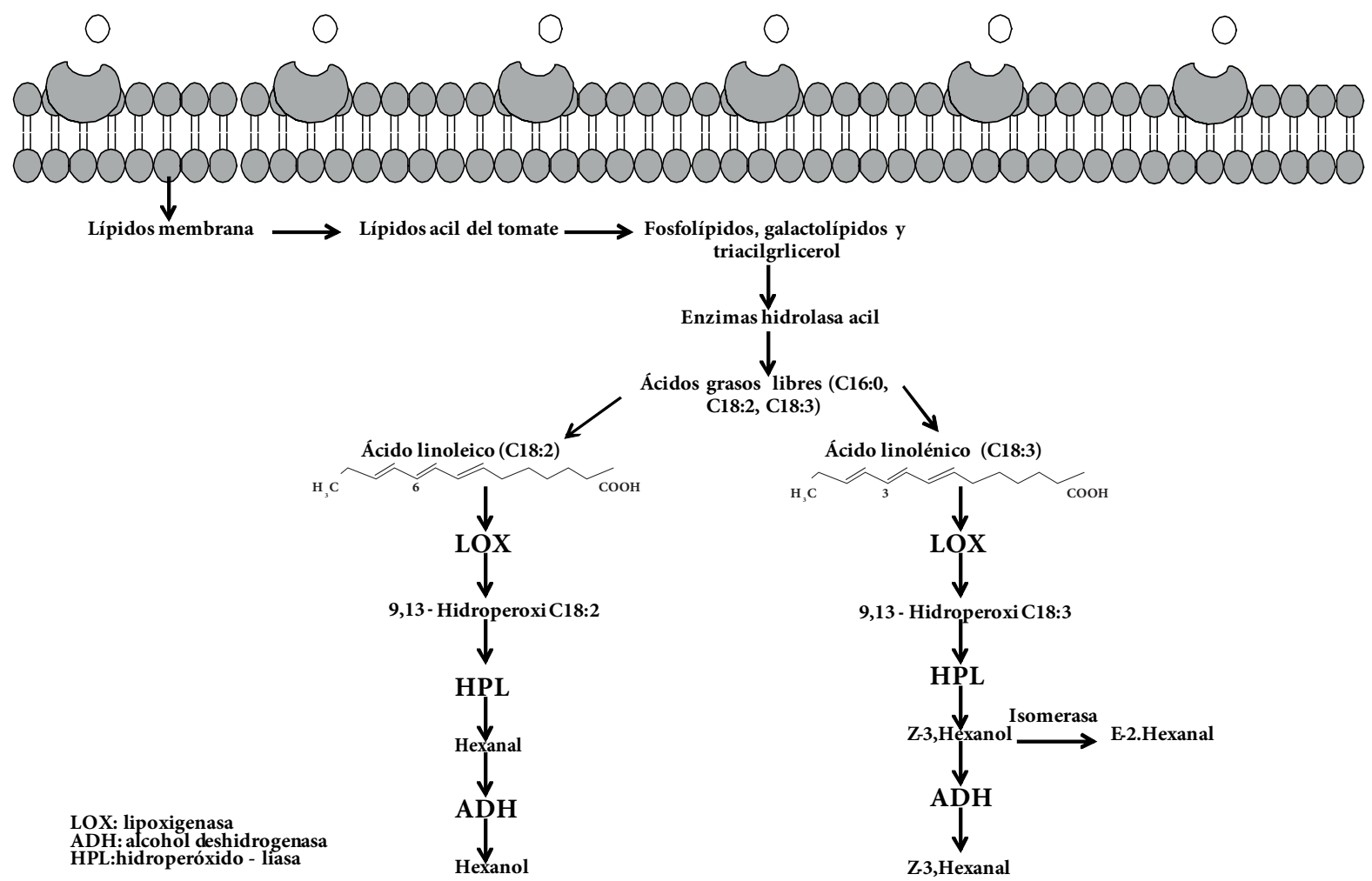

Figura 1. Formación de compuestos volátiles derivados de lípidos (LOX, lipoxigenasa; HPL, hidroperóxido-liasa; ADH, alcohol deshidrogenasa). Fuentes: Galliard et al. (1977); Riley et al. (1996). 
acciones: protonación-deshidratación del hidroperóxido, reordenamiento del catión epoxialílico en el ion oxonio, hidroxilación del ion oxonio en el hemicetal y descomposición del hemicetal en dos fragmentos de aldehídos (Prestage et al., 1999). Esta enzima está localizada en los cloroplastos y en partículas no cloroplásticas, como formas solubles en las membranas, y su actividad enzimática no cambia durante la madurez del tomate (Yilmaz, 2001b).

$\mathrm{ADH}$ es otra enzima considerada de gran importancia en el desarrollo del sabor del tomate (Baldwin et al., 2000), cuya mayor actividad se observa cuando el fruto está completamente maduro y de color rojo (Yilmaz et al., 2001c). La ADH es responsable de convertir el compuesto 3-metilbutanal formado por el ácido cetoisocaproico, en el alcohol 3-metilbutanol (Canoles et al., 2005).

Debido a que la vía de oxidación de lípidos consiste en una secuencia de enzimas (lipasa, LOX, HPL, isomerasa, ADH), como reportaron Christensen et al. (2007), se espera que haya diferentes tasas de producción de compuestos volátiles en función del número de pasos enzimáticos necesarios y de la actividad de las enzimas específicas. Los primeros compuestos volátiles formados por la acción de la HPL son los aldehídos hexanal y (Z)-3-hexenal, derivados de los ácidos linoléico y linolénico, respectivamente. La enzima isomerasa puede convertir el aldehído (Z)-3-hexenal a (E)-2-hexenal, y ambos pueden ser convertidos a los correspondientes alcoholes por la enzima ADH (Brauss et al., 1998; Anthon y Barrett, 2003). Los alcoholes generados por la actividad de la ADH son sustratos naturales para la formación de ésteres. Por la acción de la enzima alcohol aciltranferasa (AAT) se forman: hexil, (2,E)-hexenil y $(3, Z)$ hexenil éster (Salas, 2004).

Yilmaz et al. (2002a) estudiaron el efecto de la adición de las enzimas LOX y ADH, sobre la concentración de los principales compuestos volátiles del tomate rojo y verde homogenizados. Estos autores observaron mayores niveles de todos los volátiles en el grupo testigo, que en cualquier tratamiento enzimático. Las concentraciones de hexanal, cis-3-hexenal, trans-2-hexenal, y 1-penten-3-ona en tomate rojo homogeneizado suplementado con LOX, ADH o LOX $+\mathrm{ADH}$, disminuyeron durante su almacenamiento a $20^{\circ} \mathrm{C}$ por $20 \mathrm{~min}$; en cambio, no hubo diferencias significativas entre los tratamientos del tomate verde homogeneizado. En general, la concentración de compuestos volátiles fue mayor en el homogeneizado de fruta roja que en el de fruta verde. Estos resultados indican que la síntesis de la mayoría de los alcoholes y volátiles por la ruta del oxilipina es dependiente del proceso de maduración del tomate fresco.

Por su parte, Boukobza y Taylor (2002) evaluaron el efecto de adicionar ácido linoleico al fruto macerado en diferentes temperaturas y diferentes periodos de almacenamiento; ellos encontraron un incremento en la producción de volátiles (hexanal, hexenal, metilbutanal) cuando había alta concentración de ácidos grasos libres, esto derivado de la producción de volátiles por la ruta de la oxilipina (compuestos oxigenados formados por reacciones enzimáticas). Los volátiles de la ruta de la oxilipina se consideran, por mucho, los más importantes componentes de aroma de tomate (Yilmaz, 2001b), pero su papel en la percepción y en los patrones de síntesis requiere investigación adicional, para poder correlacionar el efecto puntual de la producción de estos volátiles y la aceptabilidad del producto por el consumidor.

\section{Compuestos volátiles derivados de carotenoides}

En muchos casos se desconocen las rutas metabólicas involucradas en la síntesis de algunos compuestos, como es el caso de los volátiles sintetizados a partir de carotenoides (Simkin et al., 2004). Sin embargo, si se conociera su estructura química y se correlacionara la alta producción de ciertos volátiles cuando hay acumulación de carotenoides en el fruto de tomate, se podrían predecir los posibles productos de la división oxidativa de los carotenoides y la posible relación entre los volátiles y sus posibles precursores (Cuadro 3). Por ejemplo, se considera que los volátiles del aroma de tomate, 6-metil-5-hepten-2-ona, geranil acetona y farnesil acetona, son resultado del rompimiento oxidativo de carotenoides acíclicos. De manera similar, $\alpha$-ionona, $\beta$-ionona y $\beta$-damascenona son productos probables del rompimiento oxidativo de los carotenoides cíclicos y al parecer de otros terpenoides que pueden existir en el tejido vegetal enlazados a carbohidratos (Canoles et al., 2005). En este caso (Cuadro 1), cuando las cadenas de los carotenoides $9,10,9^{\prime}$ y $10^{\prime}$ se rompen, pueden formar dos mono-aldehídos y un di-aldehído central (Lalel et al., 2004).

\begin{tabular}{|c|c|c|}
\hline Sustratos & Productos & \\
\hline Zeaxantina & 3-hidroxi-9-apo-b-caroten-9-ona (3-hidroxi-b-ionona) & 4,9-dimetildodeca-2,4,6,8,10-pentaeno- \\
\hline B-caroteno & 9-apo-b-caroten-9-ona (b-ionona) & 1,12-dial (C14 di aldehído) \\
\hline Licopeno & 6,10-dimetil-3,5,9-undecatrien-2-ona, (pseudoionona) & \\
\hline$\Delta$-caroteno & 9-apo-a-caroten-9-ona ( $\alpha$-ionona) & \\
\hline Z-caroteno & 6,10- dimetil-5,9-undecadien-2-ona (geranilacetona) & \\
\hline Fitoeno & 6,10- dimetil-5,9-undecadien-2-ona (geranilacetona) & 9-dimetildodeca-4,6,8-trienedial. \\
\hline
\end{tabular}


Los compuestos derivados de carotenoides se caracterizan por aportar un aroma frutal o floral; se pueden detectar aún cuando se encuentren en concentraciones bajas porque su umbral de olor es muy bajo, por lo que se consideran volátiles de impacto en la percepción del sabor dulce del tomate (Baldwin et al., 2008). Se ha visto que una reducción en la concentración de volátiles derivados de carotenoides, principalmente cíclicos, afecta negativamente el sabor del tomate (Vogel et al., 2010). Es de esperarse entonces que la presencia de estos volátiles en frutos maduros coincida con niveles altos de carotenoides en el fruto.

\section{Compuestos volátiles derivados de aminoácidos}

Los aminoácidos también representan una fuente importante para la formación de compuestos volátiles que contribuyen al aroma del tomate (Tieman et al., 2006). El metabolismo de los aminoácidos genera compuestos alifáticos, alcoholes ramificados, aldehídos, cetonas, ácidos y ésteres. Estos compuestos contribuyen al aroma principal de diferentes frutos, $y$ en algunos casos lo determinan (Pérez y Sanz, 2008). La biosíntesis de estos volátiles involucra principalmente tres enzimas: aminotransferasa, descarboxilasa y $\mathrm{ADH}$, íntesis en la que el aminoácido leucina es convertido a 3-metilbutanol y 3-metilbutanal (Tieman et al., 2007).

Yilmaz et al. (2002a) estudiaron el efecto de las enzimas $\mathrm{LOX}$ y $\mathrm{ADH}$ en la producción de cinco compuestos volátiles derivados de aminoácidos (acetaldehído, acetona, 2-metilbutanol, 3-metilbutanol y 2-isobutiltiazol) y de tres compuestos volátiles derivados de carotenoides (6-metil5 -hepten-ona, geranilacetona y $\beta$-ionona), en frutos de tomate rojo y verde homogenizados. Aun cuando se había reportado que los aminoácidos relacionados con compuestos volátiles se sintetizan durante la maduración de los frutos y que los trastornos del tejido no cambian su concentración, estos autores encontraron un aumento significativo en las concentraciones de acetaldehído, 6-metil-5-hepten-2ona, geranilacetona y $\beta$-ionona, durante los primeros 10 min de incubación del homogenizado de tejido de tomate rojo (testigo) y del tratado con LOX. En el tomate verde homogenizado sólo el acetaldehído mostró un incremento significativo en el tejido testigo y en el tratado con $\mathrm{ADH}$, pero menor al observado en tomate rojo. Los compuestos 6-methyl-5-hepten-2-ona y $\beta$-ionona no mostraron cambio alguno.

\section{TÉCNICAS DE EVALUACIÓN DEL AROMA DEL FRUTO DE TOMATE}

El aroma del tomate ha sido objeto de diversas investigaciones; entre las técnicas básicas utilizadas para evaluar el aroma se encuentran el análisis sensorial y la cromatografía de gases acoplada con espectrometría de masas (CG-EM), y el método de microextracción de fase sólida combinada con cromatografía de gases y espectrometría de masas (SPME-GC-MS) (Zhang et al., 2008; Tikunov et al., 2005).

\section{Análisis de volátiles en el espacio de cabeza mediante microextracción en fase sólida}

Esta técnica permite extraer y concentrar los analitos directamente en una fibra o fase estacionaria, con uno o más polímeros de extracción (Grosch, 2007). El método es de bajo costo, rápido, fácil de aplicar, y amigable con el ambiente porque no usa disolventes orgánicos. El procedimiento de microextracción en fase sólida (SPME) consta de dos etapas principales, la de extracción y la de desorción (Kataoka et al., 2000). En la primera etapa se produce la migración de analitos desde la muestra a la fibra, hasta alcanzar la situación de equilibrio; este proceso de extracción puede producirse por adsorción o absorción, en función del tipo de fibra utilizado. Para evitar que ocurran reacciones enzimáticas que generen nuevos volátiles, se agrega cloruro de sodio al tomate macerado. En la etapa de desorción, la cual puede hacerse por medio de calor, los analitos retenidos por la fibra se liberan; esta etapa se lleva a cabo cuando el SPME se acopla a un cromatógrafo de gases. La fibra se inserta en el inyector del cromatógrafo que se encuentra a una temperatura suficientemente elevada para favorecer la desorción de los analitos que entran en la columna cromatográfica para su cuantificación e identificación (Augusto et al., 2000; Reineccius, 2007).

\section{Análisis de volátiles liberados en boca y nariz}

Los volátiles liberados en la boca y la nariz al momento de la masticación, pueden variar de los obtenidos del espacio de cabeza de fruto intacto o del jugo. La liberación de los volátiles en la boca dependerá de la composición del tomate, la temperatura y el $\mathrm{pH}$ bucal, el flujo de saliva, la velocidad de masticación, la respiración y la deglución del tomate (Piggott y Schaschke, 2001). Además, algunos volátiles pueden ser generados durante la masticación, al romperse el tejido y ponerse en contacto los sustratos y las enzimas. Los compuestos presentes en el tejido antes de la masticación se podrán liberar rápidamente, mientras que los que se forman por procesos enzimáticos durante el masticado incrementarán con el tiempo de masticación (Xu y Barringer, 2009). Un estudio de los volátiles producidos y liberados durante la masticación del tomate en el espacio aéreo de la boca y la nariz, podría representar mejor la concentración y el tipo de volátiles del tomate que definen su sabor, como los evaluaron Xu y Barringer (2009) durante el proceso de masticación, mediante exhalación periódica 
Cuadro 4. Correlación entre compuestos volátiles responsables del sabor y la descripción del tomate.

\begin{tabular}{|c|c|c|}
\hline Descripción & Volátil de referencia & Referencia bibliográfica \\
\hline Dulce & $\begin{array}{l}\text { Acetona, 2-isobutiltiazol, metanol, trans-2- } \\
\text { heptanal, 6-metil-5-hepten-2-ona, } \\
\text { Geranilacetona, etanol, acetaldehído }\end{array}$ & $\begin{array}{l}\text { Tandon et al., 2003; Maul et al., 2000; } \\
\text { Abegaz et al., } 2004\end{array}$ \\
\hline Acido & $\begin{array}{l}\text { Acetona, } \beta \text {-ionona, metanol, hexanal, trans-2- } \\
\text { heptanal,geranilacetona, } 2+3 \text { - metilbutanol }\end{array}$ & $\begin{array}{l}\text { Tandon et al., 2003; Maul et al., 2000; } \\
\text { Abegaz et al., } 2004\end{array}$ \\
\hline Hierba & $\begin{array}{l}\text { Hexanal, 1-nitro-2-feniletano, } \beta \text {-ionona, } \\
\text { geranilacetona }\end{array}$ & $\begin{array}{l}\text { Berna et al., 2004; Tandon et al., 2000; } \\
\text { Maul et al., 2000; Abegaz et al., } 2004\end{array}$ \\
\hline Tomate & Cis-3-hexanal, Hexanal, geranilacetona & Tandon et al., 2000; Maul et al., 2000 \\
\hline Tomate Verde & (E)-2-hexenal, metanol, trans-2-heptanal & Berna et al., 2004; Tandon et al., 2003 \\
\hline Rancio & 3-Metilbutanol, 1-penten-3-ona & $\begin{array}{l}\text { Berna et al., 2004; et al., 2003; } \\
\text { Tandon } \text { et al., } 2000\end{array}$ \\
\hline Floral & 2-Phenyletanol & Berna et al., 2004; Tandon et al., 2000 \\
\hline Frutal & $\begin{array}{l}\text { Acetaldehído, acetona, 2-isobutiltiazol, } \\
\text { 2+3-hexenal }\end{array}$ & $\begin{array}{l}\text { Tandon et al., 2003; Abegaz et al., } \\
2004\end{array}$ \\
\hline Pungencia & $\begin{array}{l}\text { 2-isobutiltiazol, 1-penten-3-ona, 6-metil-5- } \\
\text { hepten-2-ona, trans-2-heptanal }\end{array}$ & $\begin{array}{l}\text { Tandon et al., 2003; Tandon et al., } \\
\text { 2000; Abegaz et al., } 2004\end{array}$ \\
\hline Astrigencia & $\begin{array}{l}\text { Acetona, hexanal, 6-metil-5-hepten-2-ona, } \\
\text { trans-2-heptanal }\end{array}$ & Abegaz et al., 2004 \\
\hline Metálico & 1-penten-3-ona & Tandon et al., 2003 \\
\hline
\end{tabular}

de los gases hacia un recipiente para su posterior análisis; encontraron que las concentraciones de (Z)-3-hexenal, (E)-2-hexenal, hexanal y 1-pentenona se incrementaron durante la masticación del tomate por los primeros $30 \mathrm{~s}$.

\section{Análisis sensorial}

El análisis sensorial es una disciplina científica perfectamente establecida y reglamentada (Buckenhueskes, 2007), en la que se indican metodologías que permiten relacionar un atributo del aroma con un volátil específico, éste denominado compuesto de impacto (Czerny et al., 2008). El Cuadro 4 muestra algunos estudios en los que se correlacionaron estos atributos o descripciones sensoriales con un compuesto volátil específico. En el estudio de las moléculas de interés sensorial, la estadística es una herramienta imprescindible para correlacionar las sensaciones con el análisis instrumental (Buckenhueskes, 2007). La elección del método estadístico es compleja, pues depende de si se quiere correlacionar una variable sensorial o un conjunto, o bien correlacionar un perfil obtenido en un análisis descriptivo con los datos cuantitativos cromatográficos, o cualquiera otra combinación.

\section{Otras tecnologías de evaluación del aroma}

En los últimos años se han propuesto otros sistemas que eliminan la necesidad de pruebas rutinarias de aroma para la industria alimentaria. Uno de ellos es la nariz electrónica, que es un instrumento dotado de sensores químicos y de un programa quimiométrico de reconocimiento de modelos, capaz de reconocer y comparar olores individuales o complejos (Vera et al., 2010). Este sistema se ha aplicado con éxito para pre- y postperfiles de aroma de tomate (Berna et al., 2004), y mostrado así su potencial para efectuar evaluaciones rutinarias de calidad. El reto actual es lograr que este sistema produzca lecturas de sabor similares a la percepción humana.

\section{PRINCIPALES FACTORES QUE PROMUEVEN CAMBIOS DEL AROMA Y ACEPTABILIDAD DEL FRUTO DE TOMATE}

El aroma del tomate es afectado por varios factores, como variedad o cultivar, condiciones de cultivo, estado de madurez, y condiciones de almacenamiento (Auerswald et al., 1999). Por ejemplo, se ha reportado que los tomates cultivados en campo y madurados en forma natural contienen mayor concentración de compuestos volátiles que los tomates cultivados en invernadero y madurados artificialmente (Dorais et al., 2001; Dudareva y Pichersky, 2008). A continuación se detallan los principales factores que afectan el aroma del tomate.

\section{Variedad o cultivar}

El cultivar y el genotipo tienen un efecto importante en la calidad sensorial de frutas y hortalizas (Adedeji et al., 2006; Saladié et al., 2007). En diversos estudios se han descrito diferencias significativas entre cultivares en la 
producción de volátiles, como el reportado por Brauss et al. (1998) quienes evaluaron el contenido de los volátiles hexenal, metilbutanal, metilnitrobutano, metilbutanol, hexenol, isobutiltiazol y hexanal, en cuatro variedades del tomate ('Delice,', 'Plum, 'Cherry Israeli' e 'Italiano') donde encontraron una variación considerable de estos compuestos entre variedades.

Tandon et al. (2003), al estudiar los componentes del sabor de 12 cultivares de tomate, encontraron diferencias significativas en las concentraciones de los volátiles 2-metilbutanol, 3-metilbutanol, cis-3-hexenol, geranilacetona, $\beta$-ionona, 1 -penten-3-ona y 2 -isobutiltiazol; pero no encontraron diferencias significativas en las concentraciones de hexanal, cis-3-hexenal, trans-2-hexenal, etanol, acetona y acetaldehído.

Krumbein et al. (2004) estudiaron tres cultivares de tomate ('Vanesa', 'Mickey' y 'Pronto') cosechados en estado de madurez rojo maduro; los autores encontraron diferencias entre cultivares en su contenido de compuestos volátiles, aun cuando en los tres hubo aumento de ocho compuestos volátiles (hexanal, (E)-2-heptenal, (E, E)-2,4-decadienal, 6-metil-5-hepten-2-ona, geranilacetona, 2-isobutiltiazol, 1-nitro-2-feniletano y geranial), así como reducción del salicilato de metilo durante el periodo poscosecha. El cv. 'Mickey' mostró disminución significativa en la concentración del volátil (Z)-3-hexenal, mientras que en el cv. 'Vanessa' la disminución ocurrió en el volátil (E)-2hexenal, pero esto fue sólo después de un almacenamiento por 10 d. En general, se observó la misma tendencia de incremento o decremento de volátiles responsables del sabor del tomate, independientemente de su concentración inicial.

Con respecto al desarrollo de nuevas variedades de tomate, el mejoramiento genético de esta especie se ha enfocado al rendimiento, tamaño, firmeza, resistencia a enfermedades e incremento de la vida de anaquel del fruto, sin considerar al sabor (Alonso et al., 2010). Por ejemplo, los tomates híbridos han mostrado menor concentración de volátiles de impacto como 3-metilbutanal, hexanal, cis3-hexenal, 1-hexanol y 2-isobutiltiazol, que las variedades tradicionales (Alonso et al., 2009).

Actualmente el desarrollo de nuevas variedades ya se hace tomando en cuenta el sabor (Alonso et al., 2010). Sin embargo, el mejoramiento del sabor solamente se ha dirigido hacia los contenidos de azúcares y ácidos, debido a que la producción de compuestos volátiles está regulada por un considerable número de genes involucrados en la formación del aroma, lo que ha impedido usar el mejoramiento genético convencional, además de la escasa información acerca de la relación entre la concentración, el tipo de compuestos volátiles y el aroma que imparten (Lewinsohn et al., 2001). Pero conforme se van descubriendo los genes que codifican a las enzimas involucradas en la producción de compuestos volátiles, el potencial para utilizar la ingeniería genética en la obtención de tomates con sabor mejorado, es muy prometedora (Lewinsohn et al., 2001)

\section{Estado de madurez y condiciones de almacenamiento}

Para satisfacer la demanda de tomates frescos tanto en el mercado nacional como en el de exportación, el fruto se cosecha en estado verde inmaduro o quebrante, ya que la etapa de madurez es un factor crítico en la calidad poscosecha del tomate (Yilmaz et al., 2002b). Con el fin de prolongar el tiempo de comercialización del fruto más allá del término de la estación de cosecha, éstos son almacenados en condiciones controladas, como baja temperatura, atmósfera controlada y alta humedad relativa.

El objetivo del almacenamiento controlado es disminuir la actividad biológica del producto $\mathrm{y}$, en consecuencia, reducir la pérdida de agua y de turgencia del fruto. Por otro lado, los tomates destinados al procesamiento pueden ser expuestos a altas temperaturas $\left(35 \mathrm{a} 45^{\circ} \mathrm{C}\right)$ y a altas concentraciones de oxígeno, sobre todo si se transportan a granel (Thompson et al., 2002). Con frecuencia estas prácticas inducen cambios bioquímicos indeseables porque pueden modificar los componentes responsables del aroma (González-Aguilar et al., 2010), debido a que la temperatura, la luz y el oxígeno afectan la velocidad de las reacciones de deterioro y el metabolismo normal del fruto. Por ello es que el tiempo y la temperatura de almacenamiento juegan un papel importante en la producción de aromas del tomate.

Boukobza y Taylor (2002) estudiaron el efecto del almacenamiento de tomate de la variedad 'Solairo' a cuatro temperaturas $\left(6,21,35\right.$ y $\left.45^{\circ} \mathrm{C}\right)$; encontraron niveles más bajos de volátiles a temperaturas de refrigeración $\left(6^{\circ} \mathrm{C}\right)$ que a temperatura ambiente, con excepción del isobutiltiazol, metilbutanal y metilbutanol, que incrementaron en refrigeración. Estos resultados concuerdan con Maul et al. (2000), quienes evaluaron tomates 'Solimar' y 'BHN-189' maduros (estado de madurez 5) almacenados a 20, 12.5, 10 y $5{ }^{\circ} \mathrm{C}$, durante $12 \mathrm{~d}$ de almacenamiento; encontraron que los volátiles acetaldehído, acetona y etanol, hexanal, 2-metilbutanol, 3-metilbutanol, trans-2-hexenal, trans2-heptanal, 6-metil-5-hepten-2-ona, cis-3-hexenol, 2 -isobutiltiazol y geranilacetona, aumentaron más en los frutos almacenados a $20^{\circ} \mathrm{C}$, mientras que a temperaturas por debajo de $12.5^{\circ} \mathrm{C}$ se suprimió la síntesis de volátiles y en consecuencia disminuyó el aroma del fruto.

Díaz de León-Sánchez et al. (2009) estudiaron el 
efecto del almacenamiento a $10{ }^{\circ} \mathrm{C}$ de tomate maduro (estado de madurez 5) en la producción de volátiles responsables del aroma característico de este fruto; encontraron modificaciones cuantitativas y cualitativas en la concentración de los volátiles después del sexto día de almacenamiento, y un aumento de las relaciones de 3-metilbutanal/3-metilbutanol y hexanal/hexanol, probablemente debido a una disminución en la actividad de la enzima $\mathrm{ADH}$.

\section{CONCLUSIÓN}

El conocimiento de los mecanismos y los factores que influyen en la biosíntesis de los compuestos volátiles responsables del sabor de tomate, es uno de los principales retos de hoy en día, ya que es necesario entender porqué algunas variedades de tomate no desarrollan suficiente cantidad de los volátiles responsables del sabor y resultan con menor calidad sensorial, aspecto determinante en la decisión de compra por el consumidor. Los niveles y disponibilidad de los diferentes sustratos (aminoácidos, carotenoides y ácidos grasos), de las enzimas LOX ADH, HPL y AAT, responsables de la síntesis de los volátiles, así como su respectiva expresión génica, pueden variar entre especies y entre las condiciones pre y poscosecha a las que se exponga el fruto. Por ello es importante generar información básica que ayude a entender con más claridad las rutas metabólicas que pueden activarse o suprimirse bajo las diferentes condiciones a las que se somete el fruto. La ingeniería genética y metabolómica son herramientas valiosas que podrían utilizarse para complementar el trabajo hecho hasta el momento para la mejora de nuevas variedades con mayor contenido de volátiles en el fruto.

\section{BIBLIOGRAFÍA}

Abegaz E E, K S Tandon, J W Scott, E A Baldwin, R L Shewfelt (2004) Partitioning taste from aromatic flavor notes of fresh tomato (Lycopersicon esculentum, Mill) to develop predictive models as a function of volatile and nonvolatile components. Postharv. Biol. Technol. 34:227-235.

Adedeji O, K A Taiwo, C T Akanbi, R Ajani (2006) Physicochemical properties of four tomato cultivars grown in Nigeria. J. Food Proc. Preserv. 30:79-86.

Alonso A, L Vázquez-Araújo, S García-Martínez, J J Ruiz, A A Carbonell-Barrachina (2009) Volatile compounds of traditional and virus-resistant breeding lines of Muchamiel tomatoes. Europ. Food Res. Technol. 230:315-323.

Alonso A, S García-Martínez, L Vázquez-Araújo, J J Ruiz, A A Carbonell-Barrachina (2010) Comparative post-harvest behaviour of traditional and virus-resistant Muchamiel tomatoes. J. Sci. Food Agric. 90:1056-1062.

Anthon G E, D M Barrett (2003) Thermal inactivation of lipoxygenase and hydroperoxytreinoic acid lyase in tomatoes. Food Chem. 81:275-279.

Anza M, P Riga, C Garbisu (2006) Effects of variety and growth season on the organoleptic and nutritional quality of hydroponically grown tomato. J. Food Qual. 29:16-37.

Auerswald H, P Peters, B Bruckner, A Krumbein, R Kuchenbuch (1999)
Sensory analysis and instrumental measurements of Short-term store tomatoes (Lycopersicon esculentum Mill.). Postharv. Biol. Technol. 15:323-334.

Augusto F, A L P Valente, E D Tada, S R Rivellino (2000) Screening of Brazilian fruit aromas using solid-phase microextractiongas chromatography-mass spectrometry. J. Chromatography A 873:117-127.

Ayala-Zavala J F, M A Villegas-Ochoa, G A González-Aguilar, G I Olivas (2009) Compuestos volátiles del aroma de frutos tropicales frescos cortados. In: Aspectos Nutricionales y Sensoriales de Vegetales Frescos Cortados. Ed. Trillas. México. pp:231-255.

Baldwin E A, K Goodner, A Plotto (2008) Interaction of volatiles, sugars and acids on perception of tomato aroma and flavor descriptos. J. Food Sci. 73:S294-S307.

Baldwin, E A, J W Scott, C K Shewmaker, W Schuch (2000) Flavor trivia and tomato aroma: Biochemistry and possible mechanism for control of important aroma components. HortScience 35:10131022.

Baysal T, A Demirdöven (2007) Lipoxygenase in fruits and vegetables: A review. Enzyme Microb. Technol. 40:491-496.

Berna A Z, J Lammertyn, S Saevels, C Di Natale, B M Nicolai (2004) Electronic nose systems to study shelf life and cultivar effect on tomato aroma profile. Sensors \& Actuators B Chemical 97:324333.

Beullens K, P Meszaros, S Vermeir, D Kirsanov, A Legin, S Buysens, S. N Cap, B M Nicolai, J Lammertyn (2008) Analysis of tomato taste using two types of electronic tongues. Sensors \& Actuators B Chemical 131:10-17.

Bombelli E C, E R Wright (2006) Tomato fruit quality conservation during postharvest by application of potassium bicarbonate and its effect on Botrytis cinerea. Ciencia Inv. Agraria 33:167-172.

Brauss M S, R S T Linforth, A J Taylor (1998) Effect of variety, time of eating, and fruit-to-fruit variation on volatile release during eating of tomato fruits (Lycopersicon esculentum). J. Agric. Food Chem. 46:2287-2292.

Boukobza F, A J Taylor (2002) Effect of postharvest treatment on flavour volatiles of tomatoes. Postharv. Biol. Technol. 25:321-331.

Buckenhueskes H J (2007) Industrial quality control. In: Flavours and Fragrances Chemistry, Bioprocessing and Sustainability. R G Berger (ed). Springer-Verlag. Berlin, Heidelberg. pp:303-312.

Buttery R, L Ling (1993) Volatiles of tomato fruit and plant parts: relationship and biogenesis. In: Bioactive Volatile Compounds from Plants. R Teranish, R Buttery, H Sugisawa (eds). ACS Books. Washington D.C. pp:23-34.

Buttery R G, R Teranishi, L C Ling, R A Flath, D J Stern (1988). Quantitative studies on origins of fresh tomato aroma volatiles. J. Agric. Food Chem. 36:1247-1250.

Canoles M, M Soto, R Beaudry (2005) Hydroperoxide lyase activity necessary for normal aroma volatile biosynthesis of tomato fruit, impacting sensory perception and preference. Hortscience 40:1130-1131.

Chen G, R Hackett, D Walker, A Taylor, Z Lin, D Grierson (2004) Identification of specific isoform of tomato lipoxygenase (TomloxC) involved in the generation of fatty acid-derived flavor compounds. Plant Phisiol. 136:2641-2651.

Christensen L P, M Edelenbos, S Kreutzmann (2007) Fruits and vegetables of moderate climate. In: Flavours and Fragrances Chemistry, Bioprocessing and Sustainability. R G Berger (ed). Springer-Verlag. Berlin, Heidelberg. pp:135-181.

Czerny M, M Christlbauer, A Fischer, M Granvogl, M Hammer, C Hartl, N Hernandez, P Schieberle (2008) Re-investigation on odour thresholds of key food aroma compounds and development of an aroma language based on odour qualities of defined aqueous odorant solutions. Europ. Food Res. Technol. 228:265-273.

Díaz de León-Sánchez F, C Pelayo-Zaldívarb, F Rivera-Cabrera, M Ponce-Valadeza, X Ávila-Alejandrea, F J Fernández (2009) Effect of refrigerated storage on aroma and alcohol dehydrogenase activity in tomato fruit. Postharv. Biol. Technol. 54:93-100. 
Dorais M, A P Papadopoulos, A Gosselin (2001) Greenhouse tomato fruit quality. Hort. Rev. 26:239-319.

Dudareva N, E Pichersky (2008) Metabolic engineering of plant volatiles. Curr. Op. Biotechnol. 19:181-189.

Eskin N A M (1979) Aldehydes, alcohols, and esteres: Biogenesis. In: Plant Pigments, Flavors and Textures. The Chemistry and Biochemistry of Selected Compounds. Academic Press. New York. pp:94-107.

Galliard T, J A Matthew, A J Wright, MF Fishwick (1977) The enzyme breakdown of lipids to volatile and non-volatile carbonyl fragments in distrupted tomato fruit. J. Agric. Food Chem. 28: 863- 868.

García-Sahagún M L, V Martínez-Juárez, A N Avedaño-López, M C Padilla-Sahagún, H Izquierdo-Oviedo (2009) Acción de oligosacáridos en el rendimiento y calidad de tomate. Rev. Fitotec. Mex. 32:295-301.

Gargouri M, N Ben Akacha, F Kotti, I Ben Rejeb (2008) Lipoxygenase pathway: valorization of plant oils and aroma biosynthesis. Biotechnol Agron. Soc. Environ. 12:185-202.

González-Aguilar G A, J F Ayala-Zavala, L A De La Rosa, E ÁlvarezParrilla (2010) Preserving quality of fresh-cut products using safe technologies. J. Consumer Prot. Food Safety 5:65-72.

Griffiths A, C Barry, A G Alpuche-Soli, D Grierson (1999) Ethylene and developmental signals regulate expression of lipoxygenase genes during tomato fruit ripening. J. Exp. Bot. 50:793-798

Grosch W (2007) Gas chromatography-olfactometry of aroma compounds. In: Flavours and Fragrances Chemistry, Bioprocessing and Sustainability. R G Berger (ed). Springer-Verlag. Berlin, Heidelberg. pp:363-377.

Heitz T, D R Bergey, C A Ryan (1997) A gene encoding a chloroplasttargeted lipoxygenase in tomato leaves is transiently induced by wounding, systemin, and methyl jasmonate. Plant Physiol. 114:1085-1093

Kader A A (2008) Flavor quality of fruits and vegetables. J. Sci. Food Agric. 88:1863-1868.

Kataoka H, H L Lord, J Pawliszyn (2000) Applications of solid-phase microextraction in food analysis. J. Chromatography A 880:35-62.

Krumbein A, P Peters, B Bruckner (2004) Flavor compounds and a quantitative descriptive analysis of tomatoes (Lycopersicon esculentum Mill.) of different cultivars in shorterm storage. Postharv. Biol. Technol. 35:15-28.

Lalel H J D, Z Singh, C S Tan (2004) Ripening temperatures influence biosynthesis of aroma volatile compounds in 'Kensington Pride' mango fruit. J. Hort. Sci. Biotechnol. 79:146-157.

Lewinsohn E, F Schalechet, J Wilkinson, K Matsui, Y Tadmor, K H Nam, O Amar, E Lastochkin, O Larkov, U Ravid, W Hiatt, S Gepstein, E Pichersky (2001) Enhances levels of the aroma and flavor compound $S$-Linalool by metabolic engineering of the terpenoid pathway in tomato fruits. Plant Physiol. 127:1256-1265.

Malundo T M M, R L Shewfelt, J W Scott (1995) Flavor quality of fresh tomato (Lycopersicon esculentum Mill.) as affected by sugar and acid levels. Postharv. Biol. Technol. 6:103-110.

Maneerat C, Y Hayata, K H Hiroshi, K Sakamoto, Y Osajima (2002) Application of the porapak q column extraction method for tomato flavor volatile analysis. J. Agric. Food Chem. 50:3401-3404.

Maróstica Jr M R, G M Pastore (2007) Tropical fruit flavour. In: Flavours and Fragrances Chemistry, Bioprocessing and Sustainability. R G Berger (ed). Springer-Verlag. Berlin, Heidelberg. pp:189-200.

Maul E, S A Sargent, C A Sims, E A Baldwin, M O Balaban, D J Huber (2000) Tomato flavor and aroma quality as affected by storage temperature. J. Food Sci. 65:1228-1237.

Narain N, M S Galvao, K L Santana, J J Silveira (2010) Volatile compounds in tomato-based dried products. Drying Technol. 28:232-239.

Ortiz-Serrano P, J V Gil (2010) Quantitative comparison of free and bound volátiles of two comercial tomato cultivars (Solanum lycopersicum L.) during ripening. J. Agric. Food Chem. 58:11061114.

Peach J C, A Latché, B Rest van der (2008) Genes involved in the biosynthesis of aroma volatiles and biotechnological applications.
In: Fruit and Vegetable Flavor. B Brücker, S G Wyllie (eds). CRC Press. Boca Raton, Boston, New York, Washington D.C. pp:254271.

Pérez A G, C Sanz (2008) Formation of fruit flavor. In: Fruit and Vegetables Flavor. B Brüker, S G Wyllie (eds). CRC Press. Boca Raton, Boston, New York Washington, D.C. pp:41-70.

Piggott J R, C J Schaschke (2001) Release cells, breath analysis and inmouth analysis in flavor research. Biomol. Enginer. 17:129-136.

Prestage S, R S T Linforth, A J Taylor, E Lee, J Speirs, W Schuch (1999) Volatile production in tomato fruit with modified alcohol dehydrogenase activity. J. Sci. Food Agric. 79:131-136.

Reineccius G A (2007) Flavour-isolation techniques. In: Flavours and Fragrances Chemistry, Bioprocessing and Sustainability. R G Berger (ed). Springer-Verlag. Berlin, Heidelberg. pp: 409-425.

Riley J C M, C Willemot, J E Thompson (1996) Lipoxygenase and hydroperoxide lyase activities in ripening tomato fruit. Postharv. Biol. Technol. 7: 97-107.

Saladié M, A J Matas, T Isaacson, M A Jenks, S M Goodwin, K J Niklas, R Xiaolin, J M Labavitch, K A Shackel, A R Fernie, A Lytovchenko, M A O'Neill, C B Watkins, J K C Rose (2007) A reevaluation of the key factors that influence tomato fruit softening and integrity. Plant Physiol. 144:1012-1028.

Salas J J (2004) Characterization of alcohol acytransferase from olive fruit. J. Agric. Food Chem. 52:3155-3158.

Schwab W, R Davidovich-Rikanati, E Lewinsohn (2008) Biosynthesis of plant-derived flavor compounds. The Plant J. 54:712-732.

Schwab W, P Schreier (2002) Enzymic formation of flavor volatiles from lipids. In: Lipid Biotechnology. T M Kuo, H W Gardner (eds). Marcel Dekker. New York. pp:293-318.

Simkin A, S H Shwartz, M Aulridge, M G Taylor, H J Klee (2004) The tomato carotenoid cleavage dioxygenase 1 genes contribute to the formation of the flavor volatiles $ß$-ionone, pseudoionone, and geranylacetone. The Plant J. 40:882-892.

Sinesio F, M Cammareri, E Moneta, B Navez, M Preparaio, M Causse, S Grandillo (2010) Sensory quality of fresh french and dutch market tomatoes: A preference mapping study with italian consumers. J. Food Sci. 75:S55-S67.

Sorrequieta A, G Ferraro, S B Boggio, E M Valle (2010) Free amino acid production during tomato fruit ripening: a focus on L-glutamate. Amino Acids 38:1523-1532.

Tandon K S, E A Baldwin, R L Shewfelt (2000) Aroma perception of individual volatile compounds in fresh tomatoes (Lycopersicon esculentum Mill.) as affected by the medium of evaluation. Postharv. Biol. Technol. 20: 261-268.

Tandon K S, E A Baldwin, J W Scott, R L Shewfelt (2003) Linking sensory descriptors to volatile and nonvolatile components of fresh tomato flavor. J. Food Sci. 68:2366-2371.

Taylor A J (2002) Release and transport of flavors in vivo: ysicochemical, physiological, and perceptual considerations. Compreh. Rev. Food Sci. Food Safety 1:45-57.

Taylor A J, K S Pearson, M D Hodgson, J P Landgridge, R S Linforth (2006) Effect of physiology and physical chemistry on aroma delivery and perception. Develop. Food Sci. 43:17-20.

Thompson J F, E J Mitcham, F G Michel (2002) Preparation for fresh market. In: Postharvest Technology of Horticultural Crops. A Kader (ed). 3rd ed. Univerisity of California, Agriculture and Natural Resouces 3311. pp:67-79.

Tieman D M, H M Loucas, J Y Kim, D G Clark, H J Klee (2007) Tomato phenylacetaldehyde reductases catalyze the last step in the synthesis of the aroma volatile 2-phenylethanol. Phytochemistry 68:2660-2669.

Tieman D, M Taylor, N Schauer N, A R Fernie, A D Hanson, H J Klee (2006) Tomato aromatic amino acid decarboxylases participate in synthesis of the flavor volatiles 2-phenylethanol and 2-phenylacetaldehyde. Plant Biol. 103:8287-8292.

Tikunov Y, A Lommen, C H Ric de Vos, H A Verhoeven, R J Bino, R D Hall, A G Bovy (2005) A novel approach for nontargeted data analysis for metabolomics. Largescale profiling of tomato fruit 
volatiles. Plant Physiol. 139:1125-1137.

Vera L, M Mestres, R Boqué, O Busto, J Guasch (2010) Use of synthetic wine for models transfer in wine analysis by HS-MS e-nose. Sensors \& Actuators B 143:689-695.

Vogel J T, D M Tieman, C A Sims, A Z Odabasi, D G Clark, H J Klee (2010) Carotenoid content impacts flavor acceptability in tomato (Solanum lycopersicum). J. Sci. Food Agric. 90:2233-2240.

Xu Y, S A Barringer (2009) Effect of temperature on lipid-related volatile production in tomato puree. J. Agric. Food Chem. 57:9108-9113.

Yilmaz E (2001a) The chemistry of fresh tomato flavor. Turkish J. Agric. For. 25:149-155.

Yilmaz E (2001b) Oxilipin pathway in the biosynthesis of fresh tomato volatiles. Turkish J. Biol. 25:351-360.

Yilmaz E, K S Tandon, J W Scott, E A Baldwin, R L Shewfelt (2001)
Absence of a clear relationship between lipid pathway enzymes and volatile compounds in fresh tomatoes. J. Plant Physiol. 158:1111-1116.

Yilmaz E, E A Baldwin, R I Shewfelt (2002a) Enzymatic modification of tomato homogenate and its effect on volatile flavor compounds. J. Food Sci. 67:2122-2125.

Yilmaz E, J W Scott, R I Shewfelt (2002b) Effects of harvesting maturuty and off plant ripening on the activities of lipoxygenase, hydroperoxidelyase, and alcohol dehydrogenase enzymes in fresh tomato. J. Food Biochem. 26:443-457.

Zhang Z M, D D Zeng, G K Li (2008) Study of the volatile composition of tomato during storage by a combination sampling method coupled with gas chromatography/mass spectrometry. J. Sci. Food Agric. 88:116-124. 